\title{
Drd1a-tdTomato BAC Transgenic Mice for Simultaneous Visualization of Medium Spiny Neurons in the Direct and Indirect Pathways of the Basal Ganglia
}

\author{
Jessica A. Shuen, ${ }^{1,2}$ Meng Chen, ${ }^{1,2}$ Bernd Gloss, ${ }^{3,4}$ and Nicole Calakos ${ }^{1,2,4}$ \\ ${ }^{1}$ Center for Translational Neuroscience, ${ }^{2}$ Division of Neurology, ${ }^{3}$ Neurotransgenic Core Facility, and ${ }^{4}$ Department of Neurobiology, Duke University, \\ Durham, North Carolina 27710
}

\begin{abstract}
Introduction
The expression of fluorescent proteins in defined subsets of neurons has become a powerful tool for neurobiology. One limitation of this technology is that the majority of BAC transgenic mice harnessing this technology use enhanced green fluorescent protein (EGFP) as the fluorophore. As a result, the ability to simultaneously visualize two cell populations in the same preparation is limited by the lack of lines using contrasting fluorophores. Here we report the successful application of tdTomato protein for this purpose. Historically, the creation of transgenic lines expressing non-EGFP fluorophores in defined neuronal populations has been met with only limited success, possibly be-
\end{abstract}

Received Dec. 12, 2007; revised Jan. 11, 2008; accepted Jan. 16, 2008.

This work was supported by Howard Hughes Undergraduate Research Fellowship (J.A.S.), National Alliance for Research on Schizophrenia and Depression Young Investigator Award (N.C.), Klingenstein Fellowship in the Neurosciences (N.C.), and National Institute of Neurological Disorders and Stroke K02 Independent Scientist Career Award (N.C.). The expert technical assistance of Sam Johnson of the Light Microscopy Core Facility, Jimmy Gross of the Neurotransgenic Facility, and Yuanji Pan was critical for this project and greatly appreciated. We thank Nancy Jenkins and Neal Copeland for providing recombineering reagents and Nathaniel Heintz and X. William Yang for providing the GENSAT Drd1a-EGFP and Drd2-EGFP BAC transgenic mouse lines. We greatly appreciate insightful comments from the Calakos laboratory; C. R. Gerfen; and M. Beaulieu, A. Salahpour, and A. Ramsey of the Caron laboratory; and critical review of this manuscript by A. West and B. Arenkiel. J.A.S. performed the imaging and quantification, M.C. screened the transgenic founder lines and supervised J.A.S., B.G. created the BAC transgenic vector, and N.C. designed and supervised all aspects of the experiments.

Correspondence should be addressed to Nicole Calakos, Box 2900, Duke University Medical Center, Durham, NC27710. E-mail:nicole.calakos@duke.edu. D01:10.1523/JNEUROSC1.5492-07.2008

Copyright $\odot 2008$ Society for Neuroscience $\quad$ 0270-6474/08/282681-05\$15.00/0 cause of toxicity issues, particularly for red spectral variants (Vintersten et al., 2004; Long et al., 2005). Using the regulatory promoter elements of the type la dopamine receptor gene ( $\operatorname{Drd} 1 \mathrm{a})$, we created a BAC transgenic line that identifies the direct pathway of the basal ganglia (Drd1a-tdTomato). In combination with the existing GENSAT BAC transgenic line identifying the indirect pathway (Drd2EGFP), this new transgenic line creates a novel platform for the study of basal ganglia physiology. The choice of tdTomato as a reporter may also prove useful in other circuits in which the simultaneous visualization of two defined cell populations is desired.

The basal ganglia are important for a wide range of behaviors from decision making to movement (Graybiel, 2005). Within the basal ganglia circuitry, striatal medium spiny neurons (MSNs) project either directly or indirectly to the substantia nigra pars reticulata. The so-called "direct” (or striatonigral) and "indirect” (or striatopallidal) pathways of the basal ganglia are functionally important because they exhibit opposing influences both on the activity of the output thalamic nuclei and on behavior (Albin et al., 1995; Wichmann and DeLong, 1998). Despite the well described anatomical and functional differences between the direct and indirect pathways, the ability to study the synaptic physiology of MSNs in these two pathways has been limited until recently.

BAC transgenic mice created by the
GENSAT project have enabled the visualization of pathway specificity for striatal MSNs in live preparations (Gong et al., 2003). Because in rodents the type $1 \mathrm{a}$ and 2 dopamine receptors are expressed on MSNs in the direct and indirect pathways, respectively (Le Moine and Bloch, 1995; Yung et al., 1995), expressing EGFP under these two different dopamine receptor promoters identifies MSNs in each of the pathways (Drd1a, direct pathway; Drd2, indirect pathway) (Gong et al., 2003). The ability to identify MSN cell types in live preparations is particularly useful for electrophysiological studies in which these cells are otherwise indistinguishable. Using the GENSAT mice, electrophysiological experiments have revealed differences in both intrinsic synaptic properties and disease involvement between MSNs in the two pathways, highlighting the importance of studying MSNs with reference to their pathway (Day et al., 2006; Kreitzer and Malenka, 2007; Shen et al., 2007; Surmeier et al., 2007; Ade et al., 2008).

One major limitation of the existing GENSAT lines is that both pathways cannot be simultaneously visualized because the fluorescent reporters are the same. Consequently, two separate colonies of animals are needed to obtain the data for both pathways. This approach not only increases experimental variability because experiments are separated in space, time, and genetic background, but also increases costs because of the number of animals and additional time for data acqui- 
sition that is required. To overcome this obstacle, we created a direct pathway BAC transgenic mouse line, DrdlatdTomato, that expresses a contrasting color fluorophore. By using this line in combination with the indirect pathway GENSAT line, Drd2-EGFP, it is now possible to visualize MSNs in both pathways simultaneously.

\section{Materials and Methods}

Drd1a-tdTomato BAC transgenic A BAC clone (RP23-47M2) containing the entire Drd1 a plus $185 \mathrm{~kb}$ of $5^{\prime}$ upstream sequence was used. The tdTomato cDNA (Shaner et al., 2004) with a synthetic bovine growth hormone (BGH) polyadenylation signal was inserted into the Drd1a BAC clone using homologous recombination in Escherichia coli (Lee et al., 2001). The tdTomato cDNA replaced the ATG codon plus $180 \mathrm{bp}$ of coding sequence in the Drdla gene. Recombined BACs were linearized by AscI digestion, gel purified, and electroeluted from the gel slice. After filter dialysis (VSWP02500 filter; Millipore, Billerica, MA), the BAC fragment concentration was adjusted to $1 \mathrm{ng} / \mu \mathrm{l}$ and microinjected into pronuclei of B6SJLF1 mouse oocytes (Feng et al., 2004). Six independent founder lines of Drd1a-tdTomato BAC transgenic mice were generated, and line 5 is described in this paper.

\section{Genotyping}

Genotyping of Drd1a-tdTomato transgene is done using the following primers: BGH-F (forward primer), 5-CTT CTG AGG CGG AAA GAA CC-3; dDR4 (reverse primer), 5-TTT CTG ATT GAG AGC ATT CG-3. PCR protocol is 30 cycles of $94^{\circ} \mathrm{C}$ for $30 \mathrm{~s}, 55^{\circ} \mathrm{C}$ for $30 \mathrm{~s}$, and $72^{\circ} \mathrm{C}$ for $30 \mathrm{~s}$. PCR product is $600 \mathrm{bp}$.

Tissue fixation and immunohistochemistry Tissue was minimally processed to avoid loss of endogenous fluorescent signal. Animals aged $4-6$ weeks were anesthetized by $250 \mathrm{mg} / \mathrm{kg}$ 2,2,2-tribromoethanol delivered intraperitoneally before transcardial perfusion with $4 \%$ paraformaldehyde in PBS. All animal procedures were done humanely and according to protocol approved by the Institutional Animal Care and Use Committee of Duke University. Brains were dissected and a Leica VTS- 1000 vibratome (Leica Microsystems, Bannockburn, IL) was used to section $50 \mu \mathrm{m}$ slices. Brain slices were Hoechst stained and then mounted using Fluoromount G (Southern Biotechnology Associates, Birmingham, AL). For immunostaining, slices were incubated with a 1:1000 dilution of MeCP2 rabbit polyclonal antibody (Zhou et al., 2006) (kindly provided by A. West, Duke University) or a 1:800 dilution of NeuN mouse monoclonal antibody (Millipore) in PBST (PBS with 4\% normal goat serum, $0.1 \%$ Triton X-100) for $48 \mathrm{~h}$. Slices were incubated in secondary antibodies, Alexa 488 goat anti-rabbit IgG, or Alexa 568 goat antimouse IgG (Invitrogen, Carlsbad, CA) for $1 \mathrm{~h}$ before final PBS washes and mounting.
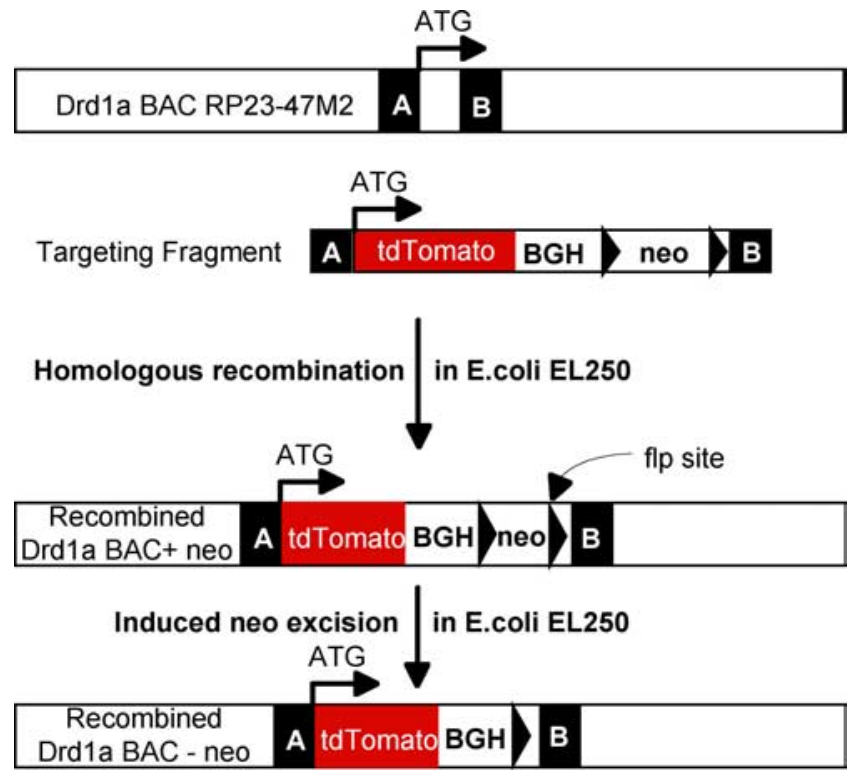

Figure 1. Modification of the Drd1a BAC clone (RP23-47M2) in E. coli by homologous recombination. Two small fragments of BAC DNA (black bars; A box and B-box) were amplified by PCR and incorporated into a target fragment to act as homologous sequences for recombination. The target fragment also contained a tdTomato cDNA followed by a flippase excisable neo gene. Through lambda Red protein-mediated homologous recombination in E. coli, the sequence (tdTomatoBGH-flp-neo-flp) flanked by the homologous fragments was inserted into the BAC clone at the desired location. The neo cassette was excised by arabinoseinduced flippase expression.

\section{Imaging and data analysis}

Images were obtained from dorsolateral striatum of 4- to 6-week-old mice using a Zeiss (Thornwood, NY) LSM510 inverted confocal with a $63 \times / 1.4$ numerical aperture $(\mathrm{NA})$ oil objective. Excitation wavelengths were 405 (Hoechst), 488 (EGFP), and 561(tdTomato) $\mathrm{nm}$. Similar results were obtained from analyses of dorsomedial and nucleus accumbens regions (data not shown). For quantification purposes, cells for analysis were first chosen solely by criteria regarding the nuclear staining images: (1) the presence of clear nuclear Hoechst staining and (2) localization within neuronal tissue (i.e., nuclei clearly in white matter or blood vessels could easily be disregarded as non-neuronal, and in fact, never had associated fluorescence). Once the population of cells for analysis was defined, the images with fluorescence from each of the fluorophores was queried for the presence of colocalizing fluorescence (see example in supplemental Fig. 2, available at www.jneurosci.org as supplemental material). In separate experiments, we determined that $74-83 \%$ of the cells selected for analysis in this manner were neuronal, depending on the marker used for immunostaining [NeuN $(74 \% ; n=480$ cells) or MeCP2 (83\%; $n=839$ cells)] (see example in supplemental Fig. 2, available at www. jneurosci.org as supplemental material). Based on this observation, an intermediate value of $80 \%$ was used to estimate the number of unlabeled cells that were neuronal. For the lowmagnification images in Figure 2, multiple images were taken using a $10 \times$ objective on an epifluorescent microscope and digitally joined to create a composite image (Photoshop
Elements; Adobe Systems, San Jose, CA). Electrophysiological rig images were taken using an Olympus (Tokyo, Japan) BX51WI microscope and a $40 \times / 0.8 \mathrm{NA}$ water-immersion objective. Images were captured using a Dage IR-1000 CCD camera (Dage-MTI, Michigan City, IN) and Scion FG Capture software (Scion, Frederick, MD).

\section{Results}

Using the same BAC clone that was used to create the GENSAT Drdla-EGFP line (RP23-47M2), we inserted the cDNA encoding tdTomato downstream of the regulatory promoter elements of the Drdla gene (Fig. 1). This construct was used to create Drdla-tdTomato BAC transgenic mice. Characterization of the transgenic progeny identified a line in which the basal ganglia pathway specificity was well preserved and similar to the GENSAT Drd1a-EGFP line (Fig. 2). A survey of nonstriatal brain expression patterns also revealed labeling similar to the GENSAT Drd1a-EGFP line (supplemental Fig. 1, available at www.jneurosci.org as supplemental material; compare with GENSAT images for dopamine receptor $\mathrm{D}_{1 \mathrm{~A}}$ identification numbers 37 and 32) and are consistent with reported expression patterns of the Drd1a gene (Weiner et al., 1991). Importantly, the intensity of the tdTomato fluorophore was adequate for visualization using a typical electrophysiological recording microscope (Fig. 3D). On a technical note, we also generated 

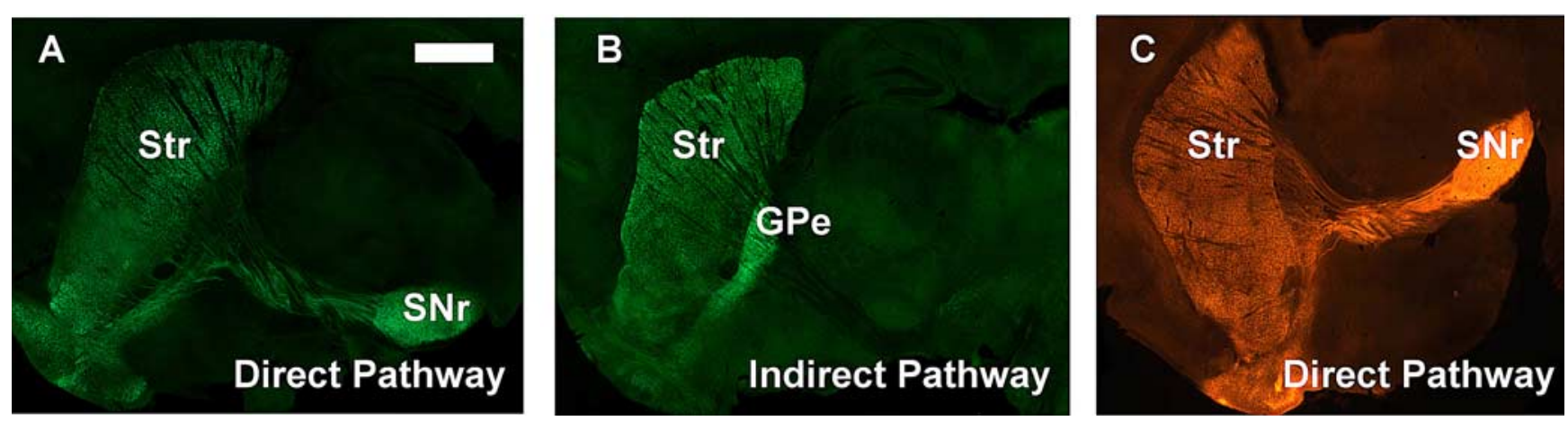

Figure 2. Low-magnification view of striatal projections demonstrates pathway specificity of BAC transgenic mice. $\boldsymbol{A}-\boldsymbol{C}$, Sagittal sections from GENSAT Drd1a-EGFP transgenic (A), GENSAT Drd2-EGFP transgenic ( $\boldsymbol{B})$, and Drd1a-tdTomato BAC transgenic ( $\boldsymbol{C}$ ) mouse lines. Fluorescence can be visualized in striatal cell bodies as well as projecting axons and terminals. Scale bar, $1 \mathrm{~mm}$. GPe, Globus pallidus externa; $\mathrm{SNr}$, substantia nigra pars reticulata; Str, striatum.

Drd1-mCherry lines, but these were found to be inferior for this purpose because the intensity of the fluorophore was lower and intracellular aggregates were prominent (data not shown).

We next crossed the GENSAT indirect pathway line (Drd2-EGFP) with our direct pathway line (Drdla-tdTomato) to determine the fidelity of these markers for identifying their respective pathways. Before this, evaluation of the integrity of the expression patterns of the GENSAT Drd1a-EGFP and Drd2-EGFP BAC transgenic lines at the cellular level was not possible because both pathway markers were the same fluorophore. Using confocal microscopy, simultaneous visualization of fluorescence resulting from the Drd1atdTomato and Drd2-EGFP transgenes in the dorsolateral striatum revealed that $<1 \%$ of cells had detectable coexpression of both fluorophores ( $n=311$ cells from 3 animals) (Fig. 3). Second, Drd1atdTomato and Drd2-EGFP expressing cells were present in approximately equal numbers (Drd1a-tdTomato, 35\%; $n=$ 1246 cells; Drd2-EGFP, 26\%; $n=588$ cells), which is in agreement with the striatal composition of direct and indirect pathway MSNs (Gerfen, 2004). Both of these findings were also observed in dorsomedial and ventral striatal regions (data not shown). The lack of colocalization between the Drd1a-tdTomato and Drd2EGFP markers demonstrates the high degree of integrity of the segregation of these markers and indicates that the positive predictive value of correctly assigning a pathway to a fluorescent cell is very high.

One practical approach to obviate the need to use two separate transgenic lines has been to study a nearby nonfluorescent cell under the assumption that it belongs to the opposite pathway as the fluorescent cells (Kreitzer and Malenka, 2007; Ade et al., 2008). However, for this alternative
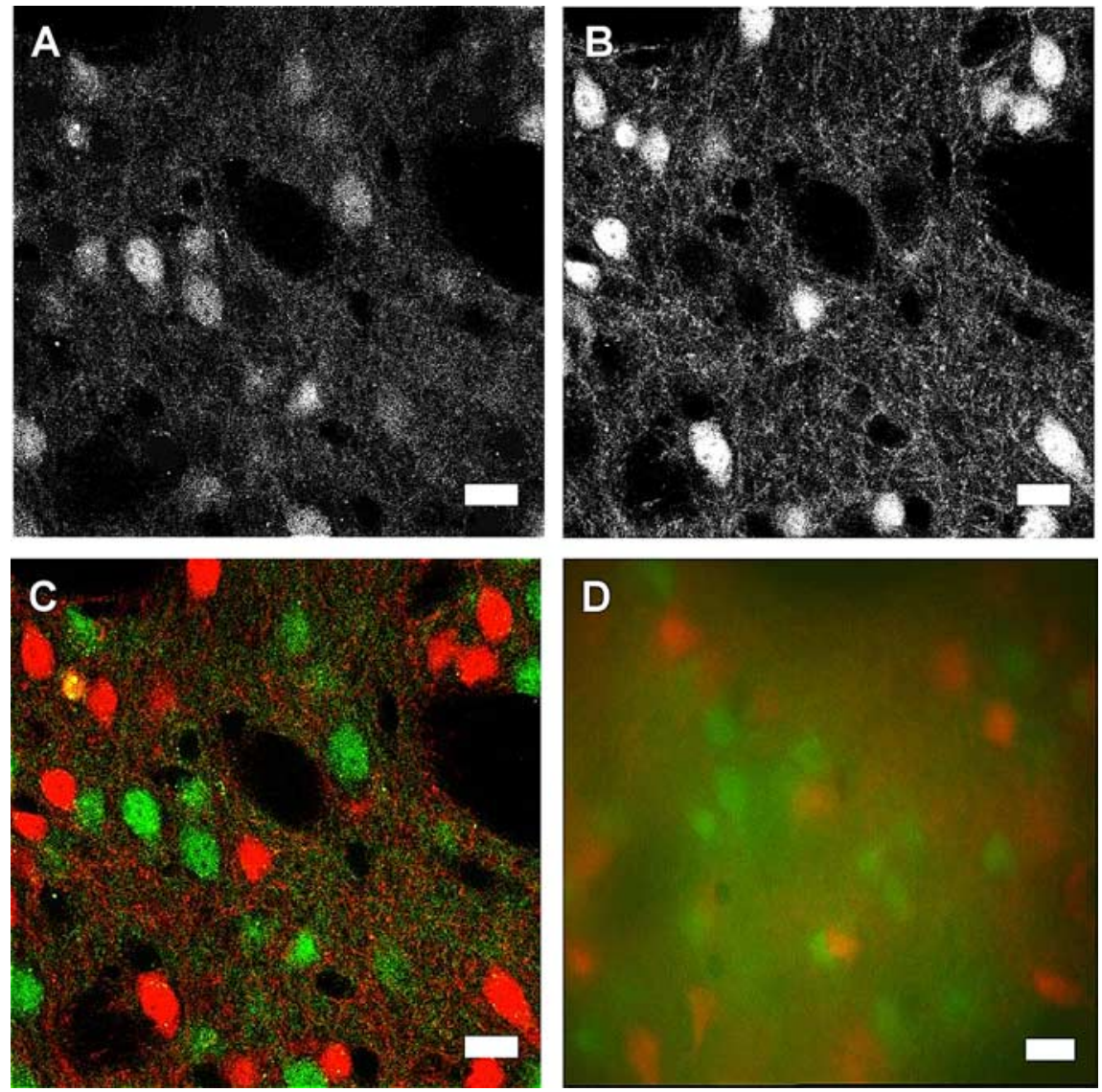

Figure 3. Accurate dual-pathway resolution at the cellular level is possible using a confocal or typical electrophysiological rig microscope. $A-D$, Striatum from Drd 1a-tdTomato ${ }^{\text {tg }} / D$ rd2-EGFP ${ }^{\text {tg }}$ mice using confocal microscopy $(63 \times$ objective, $50 \mu \mathrm{m}$ brain slice; $\boldsymbol{A}-\boldsymbol{C})$ and electrophysiological rig microscope $(40 \times$ objective, $300 \mu \mathrm{m}$ brain slice; $\boldsymbol{D})$. $\boldsymbol{A}$, EGFP. $\boldsymbol{B}$, tdTomato. $\boldsymbol{C}$, Merged image. Scale bars, $10 \mu \mathrm{m}$.

method to be accurate, the BAC transgenic lines would need to faithfully report $100 \%$ of the MSNs in a given pathway, and the imaging technique used to identify fluorescent cells would also need to be $100 \%$ sensitive in detecting "true positives." In light of these issues, we next estimated the positive predictive value of correctly assigning a nonfluorescent cell in a single transgenic line to the opposite pathway by evaluating the percentage of striatal neurons that fail to be identified by either pathway marker. Because MSNs comprise $>90 \%$ of the total striatal neurons (Kemp and Powell, 1971; Gerfen, 2004), these numbers provide a close approximation of the number of unlabeled MSNs.

Evaluating Drd1a-tdTomato ${ }^{\mathrm{tg}} / \mathrm{Drd2}$ $\mathrm{EGFP}^{\mathrm{tg}}$ mice in this manner, we found that greater than one-third (39\%) of the striatal neurons were not fluorescent for 
either of the two pathway markers (Fig. 4). To verify that the failure to detect fluorescence in all of the neurons was not unique to our new transgenic line, we also examined the striatum from animals doubly transgenic for the two GENSAT lines, Drd1a-EGFP ${ }^{\mathrm{tg}} / \mathrm{Drd2}$-EGFP ${ }^{\mathrm{tg}}$ mice. In the doubly transgenic GENSAT mice, an even greater percentage of neurons (50\%) lacked detectable fluorescent reporter expression (Fig. 4). Because our analysis measures only detectable fluorescence from the markers without further signal amplification by immunocytochemical techniques or mRNA analysis, we cannot conclude whether the unlabeled cells are a result of a failure of the transgene to be expressed, a failure of the expression level to reach a detectable threshold, or a combination of these factors. Because neither of those techniques can be used to aid detection in live preparations such as are required for slice physiology or live brain imaging, our findings are particularly relevant for these applications. As such, because a substantial fraction of MSNs fail to detectably express the pathway-specific transgenes, we have demonstrated that assigning a nonfluorescent neuron to the opposite pathway in a single transgenic animal has a high degree of error $(\sim 33 \%)$. The overall impact of this error on any given dataset will vary depending on the true magnitude of the difference in the parameter being measured for MSNs in the two pathways.

Finally, because one important application of these tools is to facilitate electrophysiological recording experiments, we tested the ability to identify fluorescent neurons reliably on a typical electrophysiological rig using acute brain slices as would be prepared for recordings (i.e., $300 \mu \mathrm{m}$ thickness) from DrdlatdTomato $^{\text {tg }} /$ Drd2-EGFP ${ }^{\text {tg }}$ doubly transgenic mice. In these experiments, a phasecontrast illumination image was taken, and all cell bodies in the focal plane were first identified. Subsequently, fluorescent images for each fluorophore (tdTomato and EGFP) were compared for signal that colocalized with the cell bodies. We identified a similar percentage of positively fluorescent cells under these conditions as with confocal microscopy. However, under these conditions, more cells appeared to colocalize with signal from both fluorophores (up to $15 \%$ ). This discrepancy with the confocal data $(<1 \%$ colocalization $)$ is likely caused by contamination from signal of nearby cells in the $z$-axis as a result of the inherent limitations imposed by the slice thickness and averaging of signal volume
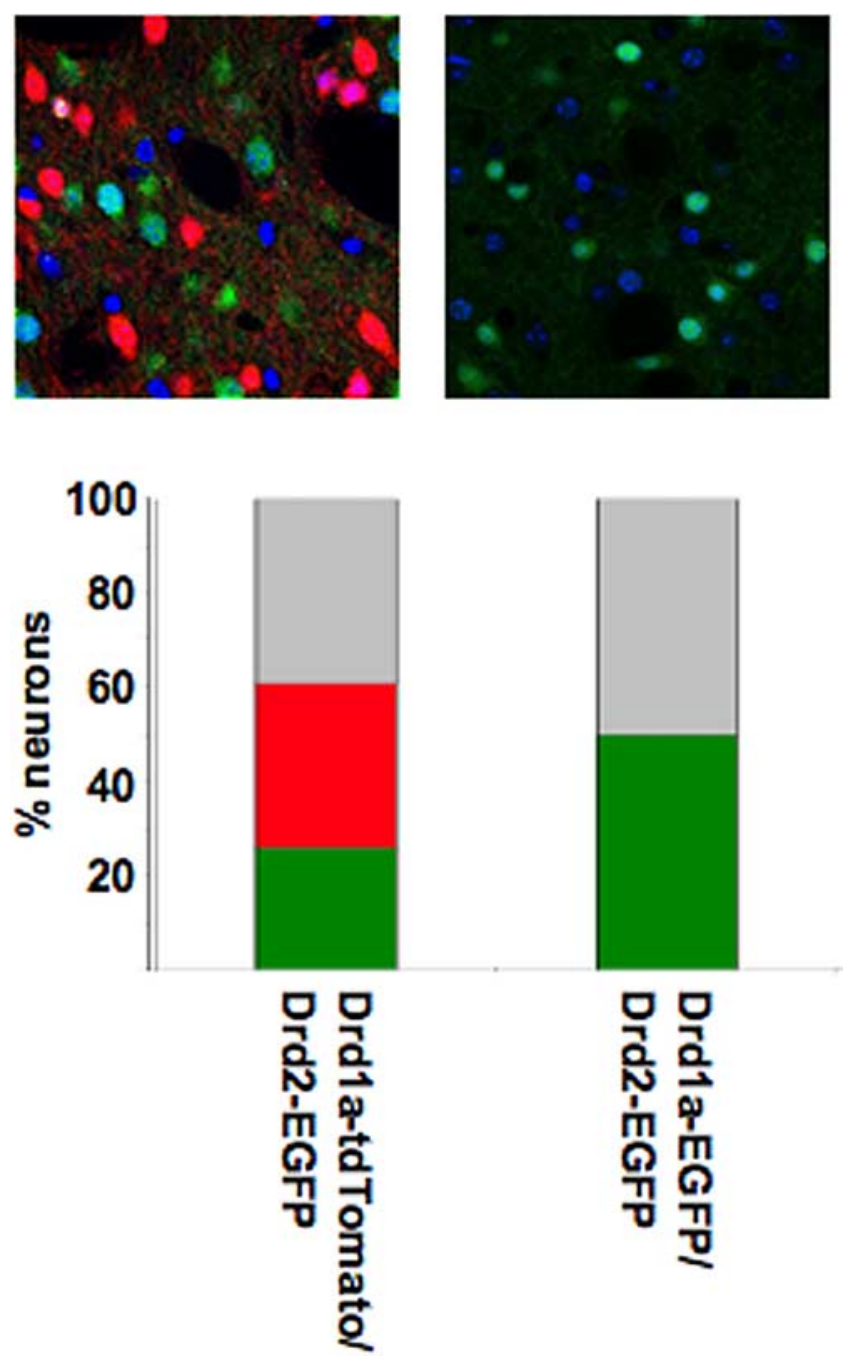

Figure 4. BAC transgenics fail to assign pathway identity to a significant fraction of striatal neurons. Confocal images of striatum from Drd1a-tdTomato ${ }^{\mathrm{tg}} /$ Drd2-EGFP ${ }^{\text {tg }}$ mice (top left) and Drd1a-EGFP ${ }^{\text {tg }} / D$ rd2-EGFP ${ }^{\text {tg }}$ mice (top right) (red, tdTomato; green, EGFP; blue, Hoechst dye). Percentage of fluorescent neurons is indicated by colored bars (green, EGFP; red, tdTomato; light gray, nonfluorescent; Drd1a-tdTomato, 35\%; $n=1246$ cells; Drd2-EGFP, $26 \% ; n=588$ cells; Drd1a-EGFP ${ }^{\text {tg }} /$ Drd2-EGFP ${ }^{\text {tg }}$ mice, $50 \% ; n=607$ cells). Unlabeled neurons were calculated by the following equation: number of nonfluorescent cells $\times 0.8$. The percentage of fluorescent neurons was determined by the following ratio: number of fluorescent cells/(number of fluorescent cells + unlabeled neurons).

with epifluorescent microscopy. This source of error can be mitigated in live recording conditions, in which the investigator has the ability to scan the $z$-axis more thoroughly and take care to ensure that the fluorescent signal truly and maximally coincides with the visualized cell body.

\section{Discussion}

In this study, we describe a new BAC transgenic mouse line that uses tdTomato as a reporter to identify MSNs in the direct pathway of the basal ganglia. This resource, for the first time, makes it possible to study live MSNs simultaneously in the direct and indirect pathways. Using this dual transgenic platform, we were also able to test several assumptions about the validity of the existing $\mathrm{BAC}$ transgenic lines as basal ganglia pathway-specific reporters. We determined that the positive predictive value of correctly assigning a pathway to a fluorescent cell is very high ( $<1 \%$ coexpression of the markers), whereas the assignment of pathway to a nonfluorescent cell has a much lower degree of accuracy.

Given these findings, by using the Drd1a-tdTomato ${ }^{\text {tg }} /$ Drd2-EGFP ${ }^{\text {tg }}$ doubly transgenic mouse platform for pathwayspecific studies of MSNs, one can avoid the additional experimental variability associated with performing experiments in two separate transgenic lines and extra costs associated with both husbandry and data acquisition time. To facilitate this approach, we recommend a simple breeding strategy in which $100 \%$ of the offspring 
are positive for both markers, further obviating the time and expense of genotyping. This is accomplished by the use of breeding pairs in which one parent is homozygous for the Drdla-tdTomato transgene and the other is homozygous for the Drd2-EGFP transgene. All of the resulting offspring are then heterozygous for both markers.

In summary, we have successfully used the red variant, tdTomato, for directed neuronal subtype expression in mouse brain. The stable, nontoxic expression of fluorophores that contrast with EGFP has been difficult historically, but is important for studies in which two cell populations are to be simultaneously visualized. Visualization of the direct and indirect pathways of the basal ganglia is one such application for this technology; however, many other potential applications exist.

\section{References}

Ade KK, Janssen MJ, Ortinski PI, Vicini S (2008) Differential tonic GABA conductances in striatal medium spiny neurons. J Neurosci 28:1185-1197.

Albin RL, Young AB, Penney JB (1995) The functional anatomy of disorders of the basal ganglia. Trends Neurosci 18:63-64.

Day M, Wang Z, Ding J, An X, Ingham CA, Shering AF, Wokosin D, Ilijic E, Sun Z, Sampson AR, Mugnaini E, Deutch AY, Sesack SR, Arbuthnott GW, Surmeier DJ (2006) Selective elimination of glutamatergic synapses on striatopallidal neurons in Parkinson disease models. Nat Neurosci 9:251-259.
Feng G, Lu J, Gross J (2004) Generation of transgenic mice. Methods Mol Med 99:255-267.

Gerfen CR (2004) The basal ganglia. In: The rat nervous system, Ed 3 (Paxinos G, ed), pp 455508. New York: Academic.

Gong S, Zheng C, Doughty ML, Losos K, Didkovsky N, Schambra UB, Nowak NJ, Joyner A, Leblanc G, Hatten ME, Heintz N (2003) A gene expression atlas of the central nervous system based on bacterial artificial chromosomes. Nature 425:917-925.

Graybiel AM (2005) The basal ganglia: learning new tricks and loving it. Curr Opin Neurobiol 15:638-644.

Kemp JM, Powell TP (1971) The structure of the caudate nucleus of the cat: light and electron microscopy. Philos Trans R Soc Lond B Biol Sci 262:383-401.

Kreitzer AC, Malenka RC (2007) Endocannabinoid-mediated rescue of striatal LTD and motor deficits in Parkinson's disease models. Nature 445:643-647.

Lee EC, Yu D, Martinez de Velasco J, Tessarollo L, Swing DA, Court DL, Jenkins NA, Copeland NG (2001) A highly efficient Escherichia colibased chromosome engineering system adapted for recombinogenic targeting and subcloning of BAC DNA. Genomics 73:56-65.

Le Moine C, Bloch B (1995) D1 and D2 dopamine receptor gene expression in the rat striatum: sensitive cRNA probes demonstrate prominent segregation of D1 and D2 mRNAs in distinct neuronal populations of the dorsal and ventral striatum. J Comp Neurol 355:418-426.

Long JZ, Lackan CS, Hadjantonakis AK (2005) Genetic and spectrally distinct in vivo imaging: embryonic stem cells and mice with widespread expression of a monomeric red fluorescent protein. BMC Biotechnol 5:20.
Shaner NC, Campbell RE, Steinbach PA, Giepmans BN, Palmer AE, Tsien RY (2004) Improved monomeric red, orange and yellow fluorescent proteins derived from Discosoma sp. red fluorescent protein. Nat Biotechnol 22:1567-1572.

Shen W, Tian X, Day M, Ulrich S, Tkatch T, Nathanson NM, Surmeier DJ (2007) Cholinergic modulation of Kir2 channels selectively elevates dendritic excitability in striatopallidal neurons. Nat Neurosci 10:1458-1466.

Surmeier DJ, Ding J, Day M, Wang Z, Shen W (2007) D1 and D2 dopamine-receptor modulation of striatal glutamatergic signaling in striatal medium spiny neurons. Trends Neurosci 30:228-235.

Vintersten K, Monetti C, Gertsenstein M, Zhang P, Laszlo L, Biechele S, Nagy A (2004) Mouse in red: red fluorescent protein expression in mouse ES cells, embryos, and adult animals. Genesis 40:241-246.

Weiner DM, Levey AI, Sunahara RK, Niznik HB, O’Dowd BF, Seeman P, Brann MR (1991) $\mathrm{D} 1$ and D2 dopamine receptor mRNA in rat brain. Proc Natl Acad Sci USA 88:1859-1863.

Wichmann T, DeLong MR (1998) Models of basal ganglia function and pathophysiology of movement disorders. Neurosurg Clin N Am 9:223-236.

Yung KK, Bolam JP, Smith AD, Hersch SM, Ciliax BJ, Levey AI (1995) Immunocytochemical localization of D1 and D2 dopamine receptors in the basal ganglia of the rat: light and electron microscopy. Neuroscience 65:709-730.

Zhou Z, Hong EJ, Cohen S, Zhao WN, Ho HY, Schmidt L, Chen WG, Lin Y, Savner E, Griffith EC, Hu L, Steen JA, Weitz CJ, Greenberg ME (2006) Brain-specific phosphorylation of MeCP2 regulates activity-dependent Bdnf transcription, dendritic growth, and spine maturation. Neuron 52:255-269. 